\author{
Voix plurielles \\ Volume 3, Numéro 2 : septembre 2006
}

Anne Marie Miraglia

\title{
La terreur, l'intertextualité et l'hybridité culturelle dans Sans Voix de Hafsa Zinaï-Koudi
}

Citation MLA : Miraglia, Anne Marie. «La terreur, l'intertextualité et l'hybridité culturelle dans Sans Voix de Hafsa Zinaï-Koudi.» Voix plurielles 3.2 (septembre 2006). 


\title{
La terreur, l'intertextualité et l'hybridité culturelle dans Sans Voix de Hafsa Zinaï-Koudi
}

\author{
Anne Marie Miraglia \\ Université de Waterloo
}

Septembre 2006

\begin{abstract}
$\mathrm{C}$
ondamnée à mort par les intégristes en Algérie, la romancière et cinéaste, Hafsa Zinaï Koudil a dû fuir son pays natal pour se réfugier alternativement en France et en Tunisie. Son œuvre, dont cinq romans publiés entre 1984 et 1997, poursuit, cependant, sa lutte pour la démocratie, pour les droits de la femme et pour la fin de l'intégrisme en Algérie. Mère de quatre enfants, Hafsa Zinaï-Koudil a dénoncé courageusement les violences faites aux femmes par les extrémistes algériens surtout pendant la guerre civile. Celle-ci a éclaté en 1992 après un coup d'état qui a interrompu le processus de démocratisation, et a privé le FIS (Front Islamique du Salut) de sa victoire électorale.

Basé sur des faits réels, le premier long-métrage de Hafsa Zinaï-Koudil « Le Démon au féminin », a été tourné clandestinement entre septembre 1992 et mars 1993 pour endiguer la montée d'un intégrisme violent après la suspension des élections et la dissolution du Front Islamique du Salut. Le film de Hafsa Zinaï-Koudil n'a pas été distribué en Algérie mais il a été projeté en 1995 à Montréal et à la Maison des Arts de Créteil, en France. "Le Démon au féminin » est son cri d'alarme contre la diabolisation de la femme par les intégristes qui inculquent, jusque dans les écoles, l'idée que les femmes sont une émanation du démon, qu'elles fraternisent avec lui. Elles sont des êtres malfaisants qui sèment la discorde. On apprend aux petites filles le mépris de leur sexe, de leur identité. Aux garçons on enseigne qu'ils sont les maîtres. Comment construire une société sur des bases aussi malsaines ? ${ }^{1}$

Hafsa Zinai-Koudil rejette l'instrumentalisation de l'Islam, le régime autoritaire, l'oppression des femmes et le bâillonnement du peuple par le FIS : «C'est [le peuple] qu'on affame, qu'on ruine, qu'on tue, comme on tue la pensée, les éveilleurs de conscience, ceux qui alertent la société » (Ibid.). Ainsi c'est au péril de sa vie que Hafsa Zinaï Koudil fait connaître les aspirations et les souffrances de son pays: "Quand on est sur leurs listes, les intégristes n'ont de cesse de vous avoir. Nos messages de démocrates ne peuvent être diffusés. C'est un piège à rats. Il est salutaire pour nous que ce message soit répercuté au-delà des frontières. » (Ibid.).

Dans ce contexte, il est évident qu'en plus de témoigner l'amour profond de Hafsa ZinaïKoudil pour l'Algérie, son œuvre romanesque et cinématographique cherchent, à l'intérieur et à
\end{abstract}


l'extérieur du pays, un appui contre la barbarie, l'abus du pouvoir et l'obscurantisme. Lancé bien avant la tragédie du 11 septembre à New York, son combat contre le terrorisme intégriste, exige qu'on s'interroge sur l'indifférence du monde occidental pour la guerre civile en Algérie et sur la fuite vers l'occident des artistes et des intellectuels algériens.

Dans Sans voix (Plon), son cinquième et dernier roman, publié en 1997, Hafsa ZinaïKoudil dévoile les menaces derrière le départ de l'exilé, son désarroi à l'étranger et la nécessité de sa participation à la lutte pour la démocratie en Algérie. Ce roman s'oppose fermement à l'isolement et à la répression en faveur de la tolérance, de la modernité et de la pluralité culturelle. Cependant, quelques protagonistes, porte-paroles de l'auteur, expriment leur méfiance à l'égard du monde politique occidental qui se montre en faveur des régimes totalitaires lorsqu'ils présentent un bénéfice économique.

[...] des Etats occidentaux ne se gênent pas d'apporter leur appui aux intégristes et travaillent diligemment à les porter au pouvoir en Algérie.

Bien sûr. Ils évaluent leur intérêt et désirent s'approprier la plus grosse part des marchés qui ne manqueraient pas de s'ouvrir dans le pays, une fois le calme revenu. Mais ce qui est incompréhensible, c'est d'entendre certains intellectuels occidentaux accorder du crédit aux thèses intégristes. Inconsciemment ou à bon escient, ils refusent l'idée d'une Algérie démocratique au sens réel du terme. Il faut bien jouer aux donneurs de leçons, non ? Et si nous accédions à la démocratie, ils seraient obligés de nous traiter d'égal à égal. N'est-ce pas trop leur demander ? $(70-71)^{2}$

Il reste, cependant, que la promotion au niveau thématique d'une ouverture sociale et politique se répercute au niveau formel de Sans voix. En dépit de l'usage de la première personne, le texte ne se borne pas à une seule voix, à une seule perspective, mais embrasse une pluralité de discours - lettres, récits emboîtés, confession et répliques théâtrales - aussi bien que des points de vue divergents provenant à la fois des victimes, femmes et hommes, comme de leurs bourreaux, les intégristes (voir le récit de Tarik, islamiste de 19 ans, 113-123). Cette coexistence d'éléments multiples en opposition est enrichie, dans Sans Voix, de références intertextuelles algériennes et occidentales. L'importance du rôle de ces références littéraires dans le texte est signalée par leur quantité et par leur récurrence. Ces références servent d'écho aux thématiques dominantes du texte tout en signalant l'universalité des problèmes qui semblent, à première vue, spécifiques à l'Algérie.

Dans le monologue intérieur que constitue ce roman, Baya, professeure de musique au Conservatoire algérien, évoque son exil à Paris où elle se réfugie pour échapper aux fanatiques religieux persuadés que la musique comme l'enseignement du français sont les instruments du diable $(99,96)$. Baya a laissé en Algérie son mari et ses enfants. Sa vie à Paris est définie par l'attente, par des promenades solitaires et par l'espoir insensé d'un visa tout aussi chimérique.

Ce roman à base autobiographique explore la situation d'un grand nombre d'intellectuels et d'artistes qui ont fui l'Algérie où il leur était « interdit de rêver, de penser, de parler, d'écrire » (69). Aussi les premiers tracts intégristes indiquaient-ils : «Celui qui nous insulte par la plume périra par la lame ». Et Houda, l'amie journaliste de Baya, reconnaît tristement : 
Ils ont tenu leur promesse en passant à l'action pour égorger à tour de bras journalistes et écrivains ainsi que tous ceux qui par leur façon de vivre disent non à l'idéologie islamiste totalitaire. Et on doit bien reconnaître $[. .$.$] que le fer de lance de leur combat reste sans conteste$ dirigé contre la femme [...] Le modèle de société qu'ils entendent imposer est basé sur la soumission totale de la femme. [...] En la soumettant, ils parviennent à imposer leur autorité sur l'ensemble de la société qui, ils le savent bien, évolue, stagne ou régresse à travers le statut donné à la femme. (69-70)

Baya est désolée de constater le nombre d'Algériennes « embrigadées » dans une « idéologie injuste et rétrograde qui, sous le couvert de la religion et d'une prétendue réappropriation de la culture arabo-islamique vise à rejeter toute idée de modernité, déclarée impie et sacrilège » (70). Ces mêmes femmes pensent que celles qui se battent pour la démocratie ne sont que « l'émanation d'un Occident corrompu » (70).

Il est clair, cependant, que le sort des réfugiés algériens en France n'est guère meilleur : avec l'interdiction de travailler, ils vivent dans la précarité et l'angoisse -- l'angoisse du rendez-vous à la préfecture comme l'angoisse d'apprendre de nouveaux assassinats. La France, comme d'autres pays occidentaux, a du mal à gérer le grand nombre de demandeurs d'asile. Les guerres, la pauvreté, les dictatures militaires qui les incitent à s'exiler dépassent les frontières de leur pays natal pour impliquer l'ensemble du monde politique international. De plus, la fuite des cerveaux, suscitée par le terrorisme et la guerre civile, est aggravée par une globalisation industrielle favorisant une immigration sélective qui prive le Tiers Monde de ses meilleurs esprits et d'un meilleur avenir.

Sans voix montre que l'exilé algérien n'a pas de choix véritable entre « l'exil ou la mort; le silence ou la mort » (23). La folie meurtrière du pays natal est juxtaposée à l'indifférence et parfois à l'agressivité du pays d'accueil. Sans ressources, sans repères, éloigné de sa famille, de ses amis, de sa profession, en somme de tout ce dont se construit une identité personnelle, l'exilé passe son temps à attendre une nouvelle vie :

Au début, cela ne va pas de soi. Le choc brutal de la liberté retrouvée me paralyse. Et je ne bouge presque pas, passant mon temps à pleurer soir et matin, abattue, le moral à plat. Un sentiment de culpabilité me tourmente; je me sens lâche d'avoir abandonné les miens. Coupable et rongée d'inquiétude. À cette torture d'ordre moral, vient s'ajouter le manque d'argent. (42)

À Paris, Baya doit « réapprendre à vivre », à marcher sans sursauter quand elle entend quelqu'un derrière elle ; à dormir, la nuit, paisiblement, sans se réveiller tremblante et terrifiée (42). Les exilés de Hafsa Zinaï-Koudil sont incapables d'oublier l'horreur - viols, enlèvements, mutilations, égorgements - et la peur sauvage se réveille avec chaque cauchemar et avec chaque sonnerie du téléphone et de la porte. Même à Paris, Baya reste hantée par l'assassinat de Saïd, un ami poète, qui n'a pas voulu prolonger son séjour à Amsterdam après sa conférence tout en sachant que les intégristes finiront par l'avoir : « Ma place est là-bas. Ma vie ou ma mort, c'est en Algérie » (16). Baya est aussi marquée par l'assassinat d'un autre poète, de toute vraisemblance Tahar Djaout, 
qui a marqué de ses mots la résistance algérienne: « Si tu te tais, tu meurs. Si tu parles, tu meurs. Alors, parle et meurs » (23). Baya ne peut oublier le suicide et la grossesse de Raja, une jeune femme enlevée par des intégristes qui ont fait d'elle leur esclave domestique et sexuelle. Elle ne peut non plus effacer de son esprit ni le meurtre de Monique, une Française mariée à un Algérien, ni la décapitation de jeunes filles désireuses de poursuivre leurs études.

Baya s'inquiète surtout de ses amies qui, comme Houria et Aïcha, continuent de se battre en Algérie. Paralysée aux jambes par les intégristes, Houria n'a pas cessé d'accueillir chez elle les enfants abandonnés, les femmes répudiées, les filles-mères rejetées et les femmes menacées de mort (37). La situation se révèle encore plus périlleuse pour Aïcha, journaliste militante, qui s'attaque ouvertement à l'intégrisme et à la dictature (38).

La nuit, je pense à Aicha qui écrivait pour rester en vie. Cloîtrée, menacée, elle s'était donné pour mission d'être témoin et mémoire, et elle consacrait tout son temps, ses jours et ses nuits, à écrire. Elle tenait la chronique des drames, vécus dans leur chair et leur sang, d'héroïnes anonymes, elle relatait leur insoutenable agonie à un auditoire suspendu à ses lèvres pour empêcher que la mort, par traîtrise, ne la surprenne. (47)

L'écriture dans Sans voix est associée à la vie et à la mort. Écrire pour s'affirmer, pour se sentir exister en se révoltant peut à la fois provoquer la mort et la déjouer : « Oui, j’écris. Pour retrouver la mémoire et la garder. Pour le sel des mots. Pour briser le cercle de l'interdit où l'on voudrait me murer. Pour déchirer le silence qui m'enferme et laisser bruire mon désir de vivre. Oui, écrire me livre et me délivre » (136). Or, il importe de remarquer que loin d'être individualistes, les efforts d'Aïcha visent le bien-être collectif et sont appuyés par son mari et son beau-père. Ce dernier observe que « le silence des indifférents autorise le crime et la trahison » (39). Cette petite phrase signale l'importance de l'écriture sur le plan national et international. En plus de nous informer, le signalement de crimes violents est aussi un appel à l'action ou du moins à une prise de position qui interroge nos mœurs et notre système de valeurs à l'échelle internationale.

Sans voix est un texte autoreprésentatif qui ne cesse de souligner l'importance de l'écriture militante et des écrivains socialement engagés. Ainsi, les intertextes mentionnés dans Sans voix sont nombreux. Ils relèvent de l'Orient et de l'Occident. Les premiers, servent surtout de modèles en faveur de la résistance algérienne, contre toute forme d'oppression, quel qu'en soit le prix; tandis que les écrivains et les intertextes occidentaux, regroupés autour de la thématique de l'engagement, de la mort et de la folie servent d'alarme pour signifier l'universalité de certains phénomènes qui dépassent les frontières.

La présence d'intertextes algériens et occidentaux dans le roman suggère non seulement la richesse intrinsèque à l'hybridité culturelle mais aussi le danger de l'épuration culturelle entreprise par le mouvement intégriste. Ainsi, professeur de français, Chérif, le mari de Baya, est parfaitement bilingue. Il apprécie et « la grandeur de la civilisation musulmane » et la contribution des Français aux connaissances humaines.

C'est ainsi qu'il eut le privilège de saisir l'incroyable rapprochement des idées et des valeurs humaines entre ces deux cultures que beaucoup s'évertuent à opposer. Il avait appris à faire la différence entre la France colonisatrice qui avait exploité son peuple durant cent trente-deux années, et la France des Droits de l'homme, celle des Sartre, Malraux ou Claude Simon... (87-88)

Loin de trahir les siens, Chérif représente une identité algérienne diamétralement opposée à 
l'amnésie historique prônée par les intégristes :

[Chérif] reconnaissait comme parties intégrantes de son algérianité les cultures arabo-musulmane et française. Il considérait que celui qui rejetait une partie de cette histoire reniait par là même son identité. Être algérien pour Chérif, c'était accepter toutes les composantes de son histoire. (88)

Ainsi le tout premier écrivain évoqué par les Algériens d'une troupe théâtrale désireuse de « rendre hommage aux artistes assassinés » (18) est le persan Omar Khayam. Ce « poète de la vie et de l'amour » (18) est l'auteur rebelle des Rubayat (Roubaï), œuvre poétique de renommée internationale, mise à l'index par la Perse du XIe siècle. Le deuxième auteur considéré est le romancier algérien, Kateb Yacine. Incarcéré lors de la répression sanglante des Algériens à Sétif le 8 mai 1945, 1' auteur de Nedjma a fait du français une arme pour revendiquer l'indépendance de l'Algérie et l'adoption des mêmes idéaux qui avaient nourri les révolutions européennes du XVIIIe et du XIXe siècles. Yacine inspire à plusieurs reprises l'écriture de Hafsa Zinaï-Koudil tout comme celle de Baya qui rédige une pièce de théâtre dans laquelle « la fille de Nedjma » se bat pour la fin de la guerre civile et pour la promotion de la femme algérienne. C'est Kateb Yacine qui a affirmé dans sa préface à La grotte éclatée, roman-poème de Yamina Mechakra, « une femme qui écrit vaut son pesant de poudre ».

Le nom de Mouloud Mammari, auteur berbère frappé de censure, suscite une brève polémique qui rappelle les difficultés de la minorité berbère en Algérie. La loi sur l'arabisation de 1991 semble viser non seulement la disparition du français mais aussi la suppression du berbère, langue parlée par un tiers de la population algérienne. Baya constate tristement que le conflit sera peutêtre toujours en vigueur en Algérie :

Est-ce une malédiction ou de la bêtise, cette inaptitude congénitale à ne pas s'entendre? Les morts sont bien morts et enterrés. Laissonsles en paix! Cette paix qu'ils n'ont pas connue de leur vivant. Estce la malédiction qui, à travers le temps et l'espace, se perpétue, réveillant la haine millénaire dans le cœur des frères, les poussant au meurtre et à la trahison? Le sort frappe au sein de cette fratrie déchirée, creusant un fleuve de sang et de larmes qui maintenant la sépare. (19)

D'autres auteurs d'origine algérienne engagés profondément dans l'évolution de l'Algérie - Mimouni, Boudjedra, Djebar, Djaout et Dib -- sont aussi évoqués tout comme la poétesse arabophone Zineb et le pied-noir Jean Sénac qui fut "le premier poète assassiné en Algérie" (18).

Ce n'est que plus tard et dans d'autres contextes situationnels qu'apparaissent les écrivains occidentaux nés en dehors de l'Algérie. Ainsi, une amie de Baya lui raconte que sa patronne, Mme Pinget, voulait qu'elle lui fasse la lecture d'un livre de Jorge Semprun. Mais la vieille femme a arrêté Abla parce que son accent « sonn[ait] bizarrement maghrébin ». On peut alors se demander si Mme Pinget, dont les propos sont fréquemment racistes, ne s'est pas trompée et de domestique et d'auteur. À l'âge de 14 ans, Jorge Semprun a quitté son pays natal, avec sa famille, pour fuir 
la guerre d'Espagne et s'exiler à Paris. En 1943, il fut envoyé au camp de concentration de Buchenwald. Son livre, L'écriture ou la vie, écrit en 1987, relate les horreurs du camp et la mort de ses amis, mais ce texte n'est pas mentionné explicitement dans Sans voix lequel reprend quelquesuns de ses thèmes. À l'instar de Semprun, la mort, l'oubli impossible et la difficulté d'écrire « la mort vécue » (Semprun) pour l'exorciser orientent à la fois l'écriture de Baya et celle d'Aïcha, son double resté en Algérie.

De même, la situation algérienne ne quitte pas l'esprit de Baya lorsqu'elle assiste à une répétition de Tartuffe, la pièce de Molière sur l'hypocrisie religieuse :

Assise dans l'ombre des gradins, j'écoute cet homme fourbe et vil prêcher le bien, lui, le mal radical! Il me rappelle, trait pour trait, les tartuffes de là-bas. Ceux-là même qui, le Livre Saint dans une main et le sabre dans l'autre, décapitent, violent, incendient dans une logique de total anéantissement. (42-43).

Le besoin d'exorciser la peur et de dénoncer la barbarie engendre l'écriture fébrile de Baya qui n'arrive pas à trouver le sommeil tant qu'elle n'a pas écrit l'horreur vécue par les Algériens et les Algériennes. Abattue par l'insomnie, la fatigue et l'éloignement, Baya craint de devenir folle. Effectivement, la folie assaille son amie Houda, journaliste poussée par sa mère, ancienne moudjahidine pour l'Indépendance algérienne, à se réfugier en France. La nuit venue, Houda fait des cauchemars et voit des apparitions menaçantes dans sa chambre. Elle demande à Baya de lire attentivement Le Horla de Guy de Maupassant qu'elle refuse de garder chez elle tant le livre la trouble : «Moi aussi, quand j'écris, il me semble que quelqu'un rôde autour de moi. Je sens comme une présence, une respiration dans ma nuque. Parfois, j'allonge la main par-dessus mon épaule pour tenter de saisir cet être invisible qui veut prendre possession de moi » (76).

Ainsi dans Sans voix, l'écriture est associée à la mort, à la folie et à la difficulté de trancher entre le réel et l'imaginaire. Pour bien décoder l'importance de cet intertexte dans Sans voix, il est important de savoir que et le narrateur du Horla et son auteur succombent à la folie. Victime d'une démence syphilitique, Guy de Maupassant tente le suicide et il est interné à Passy en 1892 où il meurt 18 mois plus tard. Le lecteur de Maupassant se souvient que le diariste dans Le Horla est obsédé par la présence d'un être invisible qui vide la carafe de son eau, brise la tige d'une rose pour aspirer son parfum et tourne les pages d'un livre resté ouvert. Ce protagoniste de Maupassant cherche à tuer l'être invisible en mettant le feu à sa propre maison, mais quand il comprend qu'il a échoué, il se dirige vers le suicide. Si je m'attarde sur ces particularités c'est parce que Sans voix revient sur cette histoire à deux reprises et en cite même des extraits (96).

Un soir, Houda et Baya s'attardent au cinéma où elles regardent l'adaptation cinématographique du roman de Balzac, Le Colonel Chabert (1932). Cet intertexte est également signifiant quoique Sans voix n'offre aucun autre détail. On se souvient que le Colonel Chabert est un homme généreux et honorable qui finit par devenir fou. Déclaré mort à la guerre, il revient chez lui et découvre que sa femme s'est remariée et refuse de le reconnaître afin de ne pas lui rendre une partie de la fortune dont elle a hérité.

Un peu plus tard, il est question d'une promenade que font Baya et Houda à l'ancienne adresse parisienne de l'auteur américain, Ernest Hemingway. Mais elles n'y trouvent aucune trace 
de l'auteur de La colline des éléphants blancs (103). La présence de cet auteur américain et de cette nouvelle dans Sans voix est tout de même éloquente. La voyageuse blonde de Hemingway, désemparée sur le quai désert d'une gare, sert de lien à la disparition mystérieuse d'Abla. Trahie par Mme Pinget qui la dénonce faussement pour vol, Abla a dû rentrer en Algérie. De plus, la tentative de suicide de Houda, internée dans une clinique psychiatrique, rappelle implicitement le suicide de Hemingway qui s'est tiré une balle dans la tête. Le traitement à l'électrochoc subi par le romancier américain à la clinique Mayo n'a fait que compromettre sa mémoire et rendre l'écriture impossible. Hemingway souffrait d'alcoolisme, de dépression et de paranoïa.

Si l'engagement social, l'exil et la mort constituent le contexte dans lequel figurent les écrivains algériens et occidentaux, il est aussi évident que les intertextes occidentaux apparaissent généralement dans le contexte de l'aliénation mentale. La folie, comme la peur du terrorisme et de l'intégrisme ne connaît pas de frontières et concerne le monde dans son ensemble. N'entendant plus la voix d'Aïcha et craignant le tarissement de sa propre voix, Baya décide de rentrer en Algérie. Et si sa rentrée a l'aspect d'un suicide, Baya va surtout « au-devant de [s]es responsabilités » (199). Elle choisit de poursuivre sa lutte pour une société tolérante, ouverte et plurielle.

\section{Notes}

${ }^{1}$ Hafsa Zinaï-Koudil citée dans l'article de Dominique Widemann « Une cinéaste algérienne qui ne se voile pas la face » Les Archives de l'Humanité. 4 avril 1995. Consulté le 23 mars 2006. <http://www.humanite.presse.fr/ journal/1995-04-04/1995-04-04-722377>

${ }^{2}$ Zinaï-Koudil, Hafsa. Sans voix. Paris: Plon, 1997. Dorénavant, toutes références à Sans voix renverront à cette édition. 